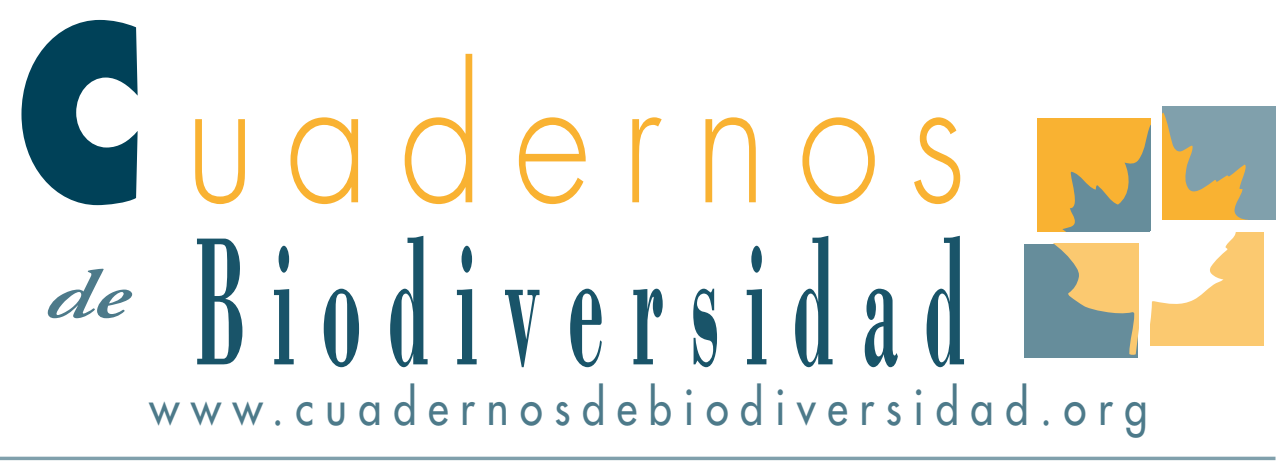

\title{
Conservación de la biodiversidad en Colombia, una reflexión para una meta: conocer y educar para conservar
}

\section{Héctor Jaime Gasca Álvarez \& Diana Torres Rodríguez ${ }^{2}$}

i Corporación Sentido Natural. Calle i 24 \# 70 F - 79. Bogotá, Colombia HJGASCA@SENTIDONATURAL.COM

2 Editorial Santillana. Carrera i i A \# 98 - 50. Piso 5. Maestría en Ciencias Biológicas. Universidad NaCional de Colombia. yenKo26@gmail.com

\section{ABSTRACT}

Given the current problems for the development of conservation projects in Colombia, a validation based on the experience of the authors is presented. This is not intended to impose a theory or philosophy, but, rather, to be a reflection on the way in which once can encourage and maintain relevant research and conservation of biological diversity in the country. It is essential to know the biodiversity of a region in order to implement conservation programs.

\section{KEY WORDS:}

Conservation biology, protected areas, natural resources, taxonomy, education.

\section{INTRODUCCIÓN}

Las acciones de las sociedades humanas sobre la naturaleza, han dejado como consecuencia un alto nivel de desaparición de especies sin precedentes en la historia (García, 2002), lo que ha generado una preocupación creciente por la conservación de la diversidad biológica. En Colombia, existe una Política Nacional de Biodiversidad (PNB), que establece un marco general para conducir y facilitar las estrategias nacionales relacionadas con el tema. Este documento, está fundamentado en tres aspectos puntuales: conservación, conocimiento y uso sostenible de la biodiversidad; siendo éste el orden de prioridad en el que se deben establecer las estrategias de manejo para el país. Recientemente el Ministerio del Medio Ambiente y Desarrollo Sostenible (2012) presentó un documento de Política de Biodiversidad (PNGISBE) abordando temas estratégicos para 
poder orientar la gestión de la biodiversidad y sus servicios ecosistémicos de manera que se puedan crear estrategias de conservación, haciendo frente al cambio ambiental de origen humano y manteniendo la resiliencia en los sistemas socio-ecológicos, contribuyendo al bienestar y la calidad de vida de los colombianos. Sin embargo, es importante cuestionarse acerca de la validez de conservar antes de conocer, y de si es realmente de ésta manera, cómo se están ejecutando las estrategias de conservación en el país.

En las últimas décadas, se ha realizado un esfuerzo para definir y conocer la biodiversidad, y entender porqué y cómo se pierde. La mayoría de autores, coinciden en definirla como la variación en la composición (componentes físicos), la estructura (disposición de los componentes) y la función (procesos e interacciones entre los componentes) de las distintas formas de vida en cualquier nivel de organización, que se manifiestan en la diversidad genética de especies, poblaciones, comunidades, biotas, ecosistemas y paisajes (Andrade et al., 1992; Andrade, 2000; Primack et al., 2001; Trombulaket al. 2004). Las acciones de conservación generalmente están enfocadas en el establecimiento de áreas protegidas, protección de especies de fauna y flora amenazadas, y la recuperación de ecosistemas degradados.

\section{EL CONOCIMIENTO DE LA BIODIVERSIDAD}

En Colombia las mayoría de las áreas protegidas, como parques nacionales y reservas biológicas, se han establecido en base su valor paisajístico, presencia de especies carismáticas o por la prestación de servicios ambientales; respondiendo a intereses sociales de preservar ecosistemas representativos sin alteraciones desencadenadas por la intervención humana (Kattan \& Naranjo, 2008) (Figs. 1 y 2). Esto evidencia que existe un conocimiento del elemento que se quiere conservar antes que una estrategia, pero además, esta estrategia de conservación se hace extensiva a grupos que no han sido necesariamente estudiados, pero que reciben la protección generada por tal reglamentación.

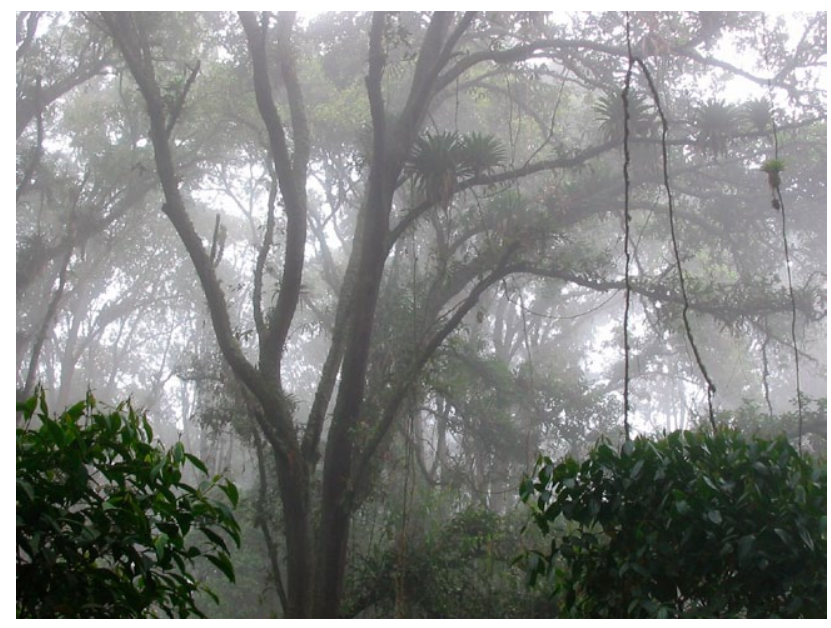

Figura 1: Bosque de Niebla, Bojacá Cundinamarca.

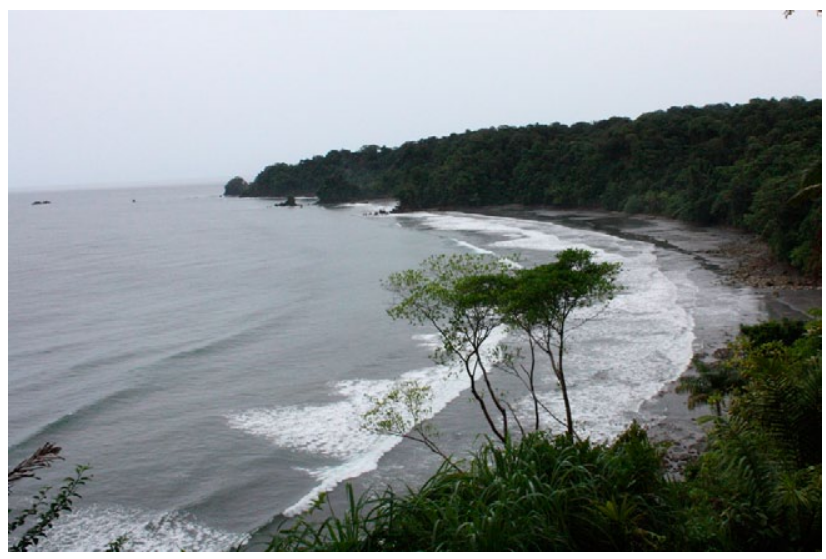

Figura 2: Chocó Biogeográfico. Colombia.

A diferencia de las estrategias de áreas protegidas, las estrategias de conservación especificas para una especie particular de flora o fauna, se generan para aquellas que cuentan con una taxonomía y ecología conocida; en un ejemplo muy puntual, los planes de conservación del Tití gris Saguinus leucopus (Günther, 1877), cuentan con un conocimiento preliminar del estado de sus poblaciones, su biología, ecología, entre otros (Rueda et al., 2011); el problema es que pocas veces se cuenta con este conocimiento.

Para generar acciones de conservación, establecimiento de planes estratégicos o cualquier otro tipo de iniciativa, es necesario saber qué es lo que se tiene, cómo y donde está, es decir, es primordial tener un conocimiento previo de los componentes de la biodiversidad, que permita obtener como mínimo una evaluación preliminar, para luego decidir sobre 
las estrategias de conservación más adecuadas. Si bien es cierto, que actualmente es una exigencia del Ministerio de Ambiente, realizar un inventario de las especies presentes en un área determinada que se planea explotar o intervenir de cualquier forma, la biodiversidad no comprende únicamente los grupos de mayor tamaño como algunas plantas, aves, mamíferos, reptiles, anfibios, entre otros. Se debe tener en cuenta que el sumatorio de estos grupos representa tan sólo una mínima porción de la estimación que se ha hecho del número de artrópodos existentes, principalmente los insectos (Andrade et al., 1996, Amat et al.,1999, Fernández et al., 2004), que son el grupo más diverso en todo el reino animal.

Por ejemplo, estudios entomológicos han encontrado que el $80 \%$ de las especies de coleópteros colectados en Panamá y un $50 \%$ de los fásmidos o insectos hoja estudiados en Costa Rica, han resultado ser especies nuevas para la ciencia, aún cuando Panamá y Costa Rica constituyen dos de las áreas mejor conocidas del Neotrópico y cubren superficies relativamente pequeñas (Primack et al., 2001). Desde este panorama, es importante pensar acerca del universo de seres conocidos de pequeño tamaño o de los ciclos y relaciones inter e intraespecíficas indispensables para la continuidad de la vida y aquellos aún sin conocer.

Las aproximaciones al conocimiento de la biodiversidad están relacionadas con la caracterización de los componentes en los niveles de especies, recursos genéticos, poblaciones, comunidades, biomas, etc. Las herramientas básicas para la evaluación de la biodiversidad, que además son indispensables para la ejecución de planes realistas de conservación y uso sostenible de los recursos bióticos, son los inventarios, censos y monitoreos, cada uno con sus propios objetivos, requisitos, criterios y fuentes de error (Stiles, 2000), lo que permite visualizar el aspecto de la biodiversidad que es importante evaluar y si se requiere, una cobertura en tiempo y espacio. Así, las listas (inventarios), conteos (censos) y seguimientos (monitoreos) delos componentes bióticos de los ecosistemas, son el primer paso para alcanzar el conocimiento de la biodiversidad.
Una opción recomendable para tener al menos un conocimiento básico, es que los estudios en biodiversidad sean extrapolables. Teniendo en cuenta que se ha empleado y se empleará muchísimo tiempo para conocer la biodiversidad de nuestro país, siendo impensable llegar a conocerla en su totalidad; el buen conocimiento de un grupo determinado, puede evitar que en los estudios omitan otros grupos de fauna y flora, como el caso de estrategias de conservación de hábitats.

Con los cambios en la manera de concebir la ciencia y el posicionamiento del conocimiento tradicional, es indispensable involucrar los estudios sociales en las estrategias de conservación. No se puede ignorar el conocimiento y valoración hacia la naturaleza de las comunidades locales, campesinas o indígenas, por lo que es necesario un trabajo de indagación de las mismas y no sólo llegar con planes que se conviertan en imposiciones para los habitantes de la zona de estudio. No se debe omitir que son ellos los actores más importantes en el proceso de conservación, ya que están directamente relacionados con su entorno natural y conocen de manera tradicional el valor de sus recursos naturales (Fig. 3).

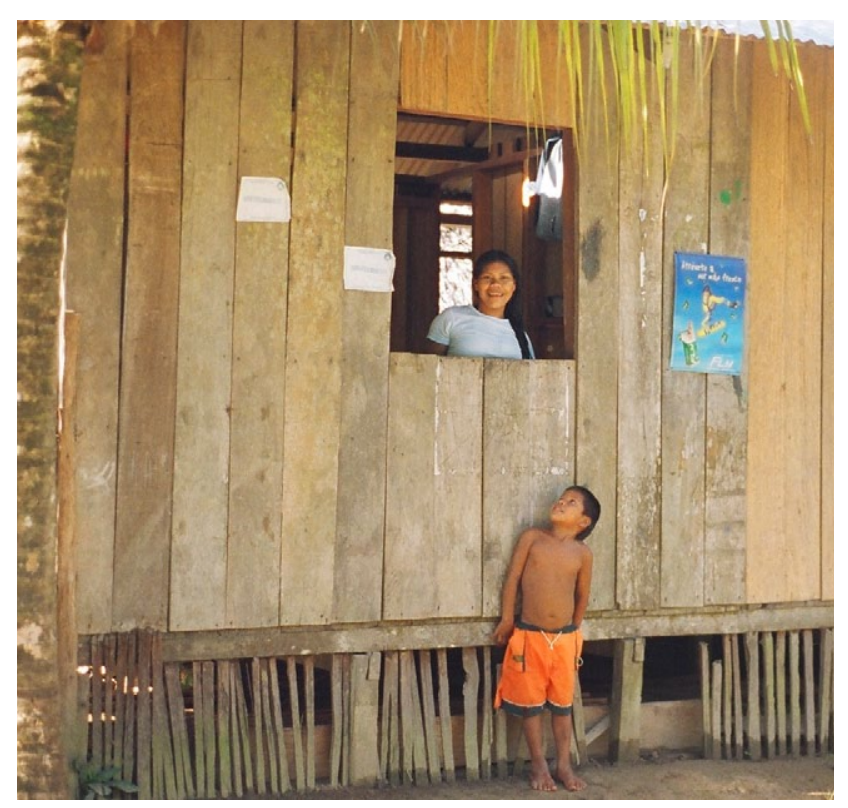

Figura 3: Comunidad Uitoto. 


\section{ESTRATEGIA INTERDISCIPLINAR}

Muchas propuestas de conservación desarrolladas en Colombia olvidan el carácter interdisciplinar de la biología de la conservación, en donde es indispensable la contribución de taxónomos, ecólogos, politólogos, sociólogos, entre otros, quienes en conjunto pueden aproximarse a una visión integrada de los problemas que competen a la biología de la conservación.

Es evidente que una estrategia de conservación es más que una metodología y que su base está en el conocimiento de la diversidad biológica y cultural. La PNB establece la necesaria participación de institutos y centros de investigación, corporaciones autónomas regionales y otras entidades, para el desarrollo de la estrategia del conocimiento de la riqueza biológica de un país, sin tener en cuenta que para dar a conocer esta riqueza es necesario educar en lo que se quiere dar a conocer, es decir, educar en lo que se quiere conservar.

El proceso de conocer la biodiversidad debe generarse en las Universidades con el establecimiento de programas eficientes de educación, basados en la investigación y con un fuerte componente taxonómico y ecológico en los diferentes niveles de la biodiversidad, usando como principal herramienta el trabajo de campo, para que el estudiante no solo construya una visión analítica sino además coherente con las realidades multidimensionales.

Para la conservación, la taxonomía es un sistema que contribuye a identificar especies cuya protección es imperativa, o a detectar áreas de alta riqueza de especies. Los patrones de diversidad se conocen fundamentalmente a través del esfuerzo de los taxónomos, sin embargo, todavía se conoce muy superficialmente la diversidad de especies en la mayoría de grupos de organismos (Primack et al., 2001)(Fig. 4).

Uno de los más graves problemas que se enfrentan en la actualidad, es el bajo número de taxónomos que realizan la descripción y clasificación de la diversidad

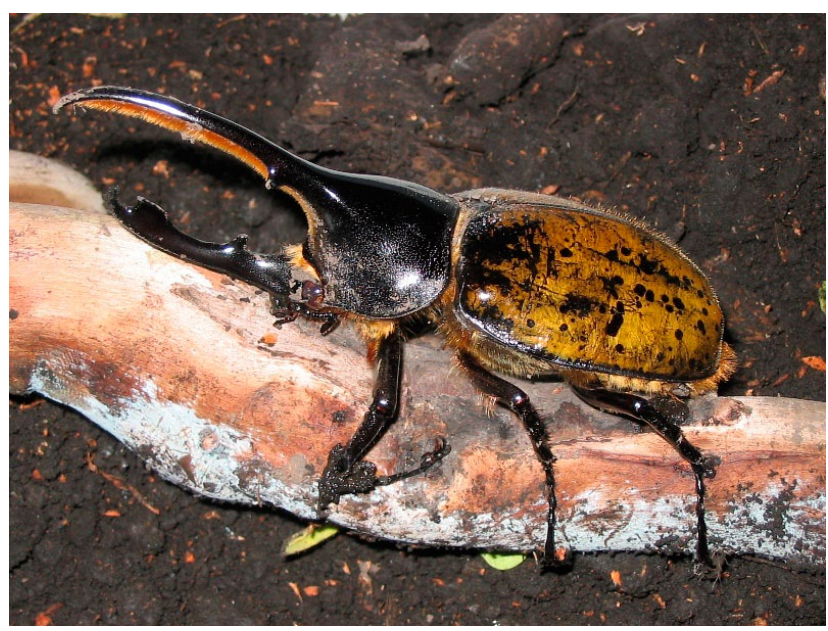

Figura 4: Dynastes hercules, especie emblemática de Colombia.

biológica; se requiere un incremento de al menos 5 veces el número de taxónomos en campo, dedicados principalmente a la descripción e identificación de las especies (Primack et al., 2001), pero esta problemática es consecuencia del bajo número de oportunidades formativas, investigadoras y laborales para estos profesionales.

La creación y promoción de programas de educación y formación para estudiantes, profesionales, técnicos y la comunidad académica en general es imprescindible para lograr aproximarse al conocimiento de la diversidad biológica. Es importante el apoyo para la ejecución de proyectos de investigación bajo los criterios y métodos adecuados, abiertos a la innovación, con el fin de consolidar un inventario nacional de biodiversidad. De igual manera, se debe tener en cuenta el papel de la educación en niveles escolares donde deben formarse actitudes necesarias para la investigación como la capacidad de síntesis, análisis, argumentación y resolución de problemas, entre otras.

Otro aspecto es el fortalecimiento de la capacidad divulgativa de corporaciones e institutos, que basados en el conocimiento generado, cumplen la función de comunicar a la comunidad el trabajo realizado y las decisiones concertadas que favorezcan tanto a grupos ecológicos como humanos. Así, las estrategias de conservación dejan de interesar sólo 
a comunidades científicas para convertirse en una actividad social, en donde es necesario que participen todas y cada una de las personas que poseen un entorno natural y que se benefician de una u otra forma de éste. Para este fin, pueden emplearse estrategias sencillas como la difusión de publicaciones pedagógicas, con un lenguaje sencillo y ameno que permita acercar y concientizar a diversas poblaciones en la conservación de los recursos naturales y generar un acercamiento de las comunidades urbanas a las comunidades rurales que les permitan comprender la necesidad de modificar algunos comportamientos propios de la sociedad occidental.

\section{ACCIONES CLARAS PARA LA CONSERVACIÓN}

La estrategia de conservación dentro del PNB incluye la reducción de los procesos que involucran pérdida de los recursos naturales por actividades antrópicas, la restauración de ecosistemas, la conservación y recuperación de poblaciones de especies silvestres amenazadas o vulnerables y finalmente la promoción de planes de conservación de especies in situ. Lamentablemente, la situación de la conservación de la biodiversidad en Colombia se torna año tras año más problemática. Diariamente se aceleran los problemas ambientales y se pierden más hábitats y poblaciones naturales;el desarrollo de actividades antrópicas ha conducido al agotamiento y la alteración de sistemas naturales reduciendo la calidad de vida de las poblaciones humanas.

Tal vez, una de las estrategias de conservación más conocidas y positivas que se han diseñado en Colombia para la protección de las comunidades bióticas funcionales, los recursos genéticos y las cuencas hidrográficas, patrimonio natural del país, es la implementación y establecimiento de "áreas protegidas" (Vásquez \& Serrano, 2009). Este concepto, que se ha replicado en todo el mundo, inspirado en la creación del Parque Nacional de Yellowstone en Estados Unidos en el año de 1872, actualmente ostenta diferentes definiciones. Tal vez la que mejor resume el verdadero significado de "área protegida" es la acepción establecida por el Convenio de Diversidad Biológica en 1994: "Área definida geográficamente designada o regulada y administrada a fin de alcanzar objetivos específicos de conservación".

La existencia de áreas naturales protegidas permite abordar la conservación in situ bajo una visión sistémica, siendo estas áreas elementos constitutivos de sistemas mayores que aseguran la continuidad de los procesos ecológicos y evolutivos que sustentan y dan origen a especies, comunidades y ecosistemas (Arango \& Díaz, 2006). La producción del conocimiento sobre representatividad de áreas protegidas en el país ha sido muy significativa en las últimas décadas, y aunque aún existen algunos vacíos de información, se han sumado esfuerzos para intentar consolidar el Sistema Nacional de Áreas Protegidas SINAP.

Uno de los logros más representativos en este aspecto, es la publicación de la obra "Las Áreas Naturales Protegidas de Colombia”, en donde se presenta un inventario completo de las áreas protegidas existentes en el país, indicando sus características biofísicas y su ubicación geográfica, sirviendo de soporte para establecer el estado actual de representatividad ecosistémica e identificando los vacíos de información en los cuales se requiere trabajar (Vásquez \& Serrano, 2009). Esta publicación es uno de los claros ejemplos que soportan la teoría que el presente ensayo pretende defender; "conocer para conservar".

Es prioritario el fortalecimiento del Sistema Nacional de Áreas Naturales Protegidas en las regiones que contienen áreas silvestres remanentes que ofrecen valores de biodiversidad y servicios ambientales importantes para la sociedad, éstas se establecen con un conocimiento básico de la biodiversidad y además permiten la continuidad de investigaciones que aporten argumentos para su permanencia.

Otra de las estrategias de conservación de la diversidad biológica, que debe estar muy ligada a la creación de áreas protegidas, es la implementación de planes concretos de conservación de especies, en 
especial de especies amenazadas o vulnerables a la extinción. Una de las problemáticas actuales tiene que ver con el desconocimiento del estado actual de las poblaciones de muchas de las especies de fauna y flora que se encuentran en áreas protegidas ya establecidas, lo que dificulta la ejecución de programas de conservación, teniendo en cuenta que los procesos de deterioro del ecosistema han continuado en varias de las áreas protegidas conocidas en el país (Calderón et al., 2005).

Sin embargo, estas acciones de conservación nunca llegarán a ser efectivas si no se cuenta, como ya se ha mencionado en líneas anteriores, con la educación y participación de las comunidades humanas locales y el apoyo de actores de carácter gubernamental como reservas municipales, alcaldías, gobernaciones, corporaciones autónomas regionales, entre otras. Las iniciativas de conservación con la participación directa de las comunidades locales han tomado fuerza en los últimos años y requieren del apoyo inmediato del gobierno, encabezados por el Ministerio del Medio Ambiente y el Departamento de Planeación Nacional. Elaborar y ejecutar un programa de conservación de áreas y/o de especies de manera exitosa, sin contar con este tipo de interacción social y sin implementar estrategias educativas y pedagógicas, es prácticamente imposible. Es muy importante generar una nueva sociedad de valores, en la que prime las necesidades de la comunidad y no el bien o lucro individual.

Finalmente, es pertinente cuestionarse si es suficiente lo que invierte el gobierno en investigación para lograr estrategias de conservación congruentes con la emergencia ambiental que enfrentamos actualmente, teniendo en cuenta que Colombia es un país megadiverso. Si bien, la biología de la conservación es una disciplina de crisis, en la que se toman decisiones para resolver problemas puntuales, se debe tener una fuerte política que permita la proyección a medio y largo plazo, con una fuerte inversión política y económica en la educación en todos sus niveles y en la investigación. Estas iniciativas, no sólo deben estar destinadas a la conservación, sino también al conocimiento primario de la biodiversidad, lo que en síntesis nos permita conocer para conservar.

\section{REFERENCIAS}

Amat, G., Andrade, G. \& Fernández, F. 1999. Insectos de Colombia. Volumen II. Academia Colombiana de Ciencias Exactas, Físicas y Naturales. Colección Jorge Álvarez Lleras. No. 13. Editora Guadalupe Ltda. Bogotá. Colombia. 433 pp.

Andrade,G.I., Gomez, R. \& Ruiz, J.P. 1992. Biodiversidad, Conservación y uso de Recursos Naturales. Colombia en el contexto internacional. Cerec - Fescol. Bogotá, Colombia. 126 pp.

Andrade, G., Amat, G. \&Fernández, F.1996. Insectos de Colombia, Estudios Escogidos. Vol. I. Academia Colombiana de Ciencias Exactas, Físicas y Naturales. Colección Jorge Álvarez Lleras No.10. Bogotá. 541 pp.

Andrade, M. 2000. Biodiversidad y Conservación de la Fauna Colombiana. Memorias I Congreso de Zoología. Facultad de Ciencias, Universidad Nacional de Colombia. Bogotá.

Arango, M. \& Díaz, J.M. 2006. Representatividad del Sistema de Áreas Protegidas de Colombia. Tomo I. En: Chaves, M.E.,Santamaría, M.(eds). Informe sobre el avance en el conocimiento y la información de la Biodiversidad 1998-2004. Instituto de Investigación en Recursos Biológicos Alexander von Humboldt. Bogotá Colombia D.C. 2 Tomos. p. 271-285.

Calderón, E., Galeano, G. \& García, N. 2005. Libro Rojo de las Plantas de Colombia. Volumen 2. Ministerio del Medio Ambiente. Instituto de Investigación en Recursos Biológicos Alexander von Humboldt. Universidad Nacional de Colombia. 454 pp.

Fernández, F., Andrade, M.G. \& Amat, G. 2004. Insectos de Colombia Volumen III. Universidad Nacional de Colombia (sede Bogotá). Instituto de Investigación en Recursos Biológicos Alexander von Humboldt. Bogotá. 602 pp.

García, R. 2002. Biología de la conservación: conceptos y prácticas. Instituto Nacional de Biodiversidad (INBio), Santo Domingo, Heredia, Costa Rica. 166 pp.

Kattan, G.H. \& Naranjo, L.G. 2008. Regiones biodiversas: herramientas para la planificación de sistemas regionales de áreas protegidas. Fundación EcoAndina, WCSColombia, WWF-Colombia, Cali, Colombia.223 pp.

Ministerio Del Medio Ambiente y Desarrollo Sostenible. 2012. Política nacional para la gestión integral de la Biodiversidad y sus Servicios Ecosistémicos (PNGISBE). Colombia. 134 pp. 
Primack, R., Rozzi, R., Feinsinger, P., Dirzo, R. \& Massardo, F. 2001.Fundamentos de conservación biológica: Perspectivas Latinoamericanas. Fondo de Cultura Economica, México D.F.797 pp.

Rueda, L.E, Sánchez, I.M. \& Aguirre J.2011. Estrategias de manejo y conservación del tití gris (Saguinus leucopus) en el área de influencia del Proyecto Hidroeléctrico Porce III, Antioquia, Colombia. Guía de Campo. Instituto de Ciencias Naturales, Universidad Nacional de Colombia. Bogotá. 140 pp.

Stiles, F.G. 2000. Inventarios, Censos y Monitoreo de la Diversidad Biológica. Memorias I Congreso de Zoología. Facultad de Ciencias, Universidad Nacional de Colombia. Bogotá.
Trombulack, S.C., Omland, K.S., Robinson, J.A., Lusk, J.J., Fleischner, T.L., Brown, G. \& Domroese, M. 2004. Principles of Conservation Biology: Recommended Guidelines for Conservation Literacy from theEducation Committee of the Society for Conservation Biology. Conservation Biology. 5: 1180-1190.

Vasquez, V.H. \& Serrano, M. 2009. Las Áreas Naturales Protegidas de Colômbia. Conservación Internacional - Fundación Biocolombia. Primera Edición. Bogotá, Colombia. 696 pp. 